Specific polymorphisms in the gene IDE (insulin-degrading enzyme) are associated with late-onset Alzheimer disease (LOAD), according to a new study from the Mayo Clinic College of Medicine, Jacksonville, USA. "Our results provide evidence for the existence of functional variants in IDE that influence LOAD risk," says lead author Nilufer Ertekin-Taner.

LOAD is the most common cause of dementia in the elderly, and the genetics of the disease have been investigated in previous studies. "Despite intensive research efforts ... there is a substantial genetic component of LOAD that remains unexplained," explains Ertekin-Taner. Currently, the apolipoprotein $\mathrm{E} \varepsilon 4$ allele is the only known genetic risk factor for LOAD.

Zou et al. investigated whether gene expression levels in the brain are a useful endophenotype for LOAD and can influence the risk of Alzheimer disease (AD). The researchers analyzed data from a published genome-wide association study by Carrasquillo et al. that screened the genomes of 970 people with LOAD and 1,495 controls to identify genes associated with LOAD. Zou and colleagues measured messenger RNA (mRNA) levels for 12 candidate LOAD-related genes in the cerebellum of 200 patients in whom the disease had been diagnosed between 60 and 80 years of age and also confirmed at autopsy. The investigators identified three IDE cis-single nucleotide polymorphisms (SNPs) that were associated significantly with IDE expression levels. They found that one cis-SNP, rs7910977, showed an association at genome-wide significance, and that presence of the minor allele of this SNP led to a twofold increase in IDE mRNA levels. Finally, they observed that the rs 7910977 SNP was significantly associated with higher levels of IDE transcript levels and with reduced LOAD risk in seven independent case-control series comprising 2,280 patients with $\mathrm{AD}$ and 2,396 controls.

Ertekin-Taner notes that Carrasquillo et al. have recently shown that the rs6583817 SNP, which lies in a conserved region of IDE and has strong linkage disequilibrium with rs7910977, is associated with increased IDE expression and reduced LOAD risk and amyloid- $\beta$ ( $A \beta$ ) levels. "Collectively, these findings provide evidence for the presence of functional variants in IDE that influence its expression in the brain and modify both LOAD risk and A $\beta$ levels," she adds.

John Hardy, a geneticist from the Institute of Neurology at University College London, UK who specializes in neurological disease, is more circumspect: "is IDE an AD gene? It may or may not be, and this study is just one more bit of evidence in the mix." Hardy warns against interpreting expression data from disease tissue and argues "it is much stronger to develop an understanding of expression in control brain where you are not confounded by all the complexities of the disease".

"It will be important to study the whole genome and transcriptome jointly to identify variants [that influence LOAD risk]," concludes Ertekin-Taner, who hopes that identifying disease susceptibility variants in large cohorts may help clinicians predict risk of disease in preclinical populations.

\title{
Katrina Ray
}

Original article Zou, F. et al. Gene expression levels as endophenotypes in genome-wide association studies of Alzheimer disease. Neurology 74, 480-486 (2010)

Further reading Carrasquillo, M. M. et al. Genetic variation in $P C D H 11 X$ is associated with susceptibility to late-onset Alzheimer's disease. Nat. Genet. 41, 192-198 (2009) | Carrasquillo, M. M. et al. Concordant association of insulin degrading enzyme gene (IDE) variants with IDE mRNA, $A \beta$, and Alzheimer's disease. PLoS One 5, e8764 (2010) 